\title{
BMJ Open Cross-sectional study comparing cognitive function in treatment responsive versus treatment non- responsive schizophrenia: evidence from the STRATA study
}

Edward Millgate (D) ,' Eugenia Kravariti, ${ }^{1,2}$ Alice Egerton, ${ }^{1,2}$ Oliver D Howes, ${ }^{1,2}$ Robin M Murray, ${ }^{1,2}$ Laura Kassoumeri, ${ }^{1,2}$ Jacek Donocik, ${ }^{1}$ Shôn Lewis, ${ }^{3,4}$ Richard Drake (D) , ,3 Stephen Lawrie, ${ }^{3}$ Anna Murphy, ${ }^{3,4}$ Tracy Collier, ${ }^{1}$ Jane Lees, ${ }^{3,4}$ Charlotte Stockton-Powdrell, ${ }^{3}$ James Walters, ${ }^{6}$ Bill Deakin, ${ }^{3}$ James MacCabe ${ }^{1,2}$

To cite: Millgate $\mathrm{E}$, Kravariti $\mathrm{E}$ Egerton A, et al. Crosssectional study comparing cognitive function in treatment responsive versus treatment non-responsive schizophrenia: evidence from the STRATA study. BMJ Open 2021;11:e054160. doi:10.1136/ bmjopen-2021-054160

- Prepublication history and additional supplemental material for this paper are available online. To view these files, please visit the journal online (http://dx.doi.org/10.1136/ bmjopen-2021-054160).

Received 03 June 2021 Accepted 04 October 2021

Check for updates

(c) Author(s) (or their employer(s)) 2021. Re-use permitted under CC BY. Published by BMJ.

For numbered affiliations see end of article.

Correspondence to Dr James MacCabe; james.maccabe@kcl.ac.uk

\section{ABSTRACT}

Background $70 \%-84 \%$ of individuals with antipsychotic treatment resistance show non-response from the first episode. Emerging cross-sectional evidence comparing cognitive profiles in treatment resistant schizophrenia to treatment-responsive schizophrenia has indicated that verbal memory and language functions may be more impaired in treatment resistance. We sought to confirm this finding by comparing cognitive performance between antipsychotic non-responders (NR) and responders (R) using a brief cognitive battery for schizophrenia, with a primary focus on verbal tasks compared against other measures of cognition.

Design Cross-sectional.

Setting This cross-sectional study recruited antipsychotic treatment $R$ and antipsychotic NR across four UK sites. Cognitive performance was assessed using the Brief Assessment of Cognition in Schizophrenia (BACS). Participants One hundred and six participants aged 18-65 years with a diagnosis of schizophrenia or schizophreniform disorder were recruited according to their treatment response, with $52 \mathrm{NR}$ and $54 \mathrm{R}$ cases. Outcomes Composite and subscale scores of cognitive performance on the BACS. Group (R vs NR) differences in cognitive scores were investigated using univariable and multivariable linear regressions adjusted for age, gender and illness duration.

Results Univariable regression models observed no significant differences between $R$ and NR groups on any measure of the BACS, including verbal memory $(B=-1.99$, $95 \% \mathrm{Cl}-6.63$ to $2.66, \mathrm{p}=0.398$ ) and verbal fluency ( $B=1.23,95 \% \mathrm{Cl}-2.46$ to $4.91, \mathrm{p}=0.510$ ). This pattern of findings was consistent in multivariable models.

Conclusions The lack of group difference in cognition in our sample is likely due to a lack of clinical distinction between our groups. Future investigations should aim to use machine learning methods using longitudinal first episode samples to identify responder subtypes within schizophrenia, and how cognitive factors may interact within this.

Trail registration number REC: 15/L0/0038.

\section{Strengths and limitations of this study}

- The study examined cognitive performance in a relatively large and multicentre sample of antipsychotic responders and non-responders.

- Cognition was assessed with the Brief Assessment of Cognition in Schizophrenia, a reliable and brief test battery specifically designed for schizophrenia.

- The lack of significant group differences in cognition between antipsychotic responders and nonresponders may reflect limited clinical separation between these groups.

\section{INTRODUCTION}

Up to one-third of patients with a schizophrenia diagnosis have inadequate symptomatic improvement despite having at least two antipsychotic drugs, one being a second-generation antipsychotic excluding clozapine, at adequate doses and duration (4-6 weeks; National Institute of health and Care Excellence (NICE) guidelines) ${ }^{1}$ and are termed treatment resistant (TRS). ${ }^{23}$ Almost all guidelines recommend the antipsychotic clozapine in $\mathrm{TRS}^{4}$ with earlier clozapine treatment associated with better outcomes. ${ }^{5-8}$ There is increasing evidence that TRS may represent a distinct subtype in schizophrenia. ${ }^{10}$ Most treatment resistant cases exhibit antipsychotic non-response (NR) from the first episode with this observed in $70 \%-84 \%$ of patients. ${ }^{3}{ }^{11}$ An earlier age of onset has also been consistently associated with antipsychotic treatment resistance, ${ }^{12-16}$ suggesting that TRS and NR may be associated with neurodevelopmental impairment. Identifying these underlying factors associated with antipsychotic treatment resistance 
in schizophrenia is therefore important for improving prediction and early treatment of NR and TRS.

Cognitive impairment in schizophrenia may provide some insight into antipsychotic treatment response. Performance on tasks of verbal memory has often been reported to be impaired in schizophrenia samples, ${ }^{17}$ those prior to medication initiation ${ }^{18}$ and at first episode. ${ }^{19}{ }^{20}$ Indeed, impairments in verbal memory and language functions have also been reported in unaffected first-degree relatives of schizophrenia patients relative to healthy controls. ${ }^{21}{ }^{22}$ Verbal memory and verbal working memory functions have also been reported to show a protracted maturation into adulthood, with impairments in these functions observed in both early and late schizophrenia. ${ }^{23}$ This suggests a possibility of a genetic and cognitive continuum of risk in schizophrenia, which increases from controls to first-degree relatives, to treatment responsive schizophrenia. A broader hypothesis is that treatment resistance is aetiologically continuous with treatment responsive schizophrenia but occupies a more exaggerated position on a continuum of neurodevelopmental liability.

In a recent meta-analysis comparing mostly crosssectional studies of treatment resistant cases and responders, TRS cases exhibited greatest cognitive impairments on tasks of verbal memory and learning $(d l=-0.59$, $\mathrm{p}<0.001)$ and language functions $(d l=-0.53, \mathrm{p}<0.001)$, with smaller but still statistically significant impairments in tasks across other cognitive domains, relative to their responder counterparts. ${ }^{24}$ However, this meta-analysis included an array of cognitive tasks, many with long test duration and stringent training requirements for raters. Short and comprehensive measures of cognitive performance may aid in the detection of neuropsychological differences between antipsychotic responders (R) and non-responders (NR), while also being cost-effective. The Brief Assessment of Cognition in Schizophrenia (BACS) ${ }^{25}$ was originally developed to be an easily administrable, brief, test battery that efficiently and specifically assesses cognitive deficits in schizophrenia cases. The measures included in the battery correspond to several cognitive domains with established deficits in schizophrenia, executive functions, ${ }^{2627}$ working memory, ${ }^{2829}$ motor/processing speed ${ }^{30}$ verbal memory, ${ }^{31}{ }^{32}$ verbal fluency ${ }^{33} 34$ and attention. ${ }^{35}$ If observable differences between antipsychotic $\mathrm{R}$ and NR are identified, this would further improve our understanding of cognitive factors implicated in the aetiology of antipsychotic response. Likewise, this would raise the possibility for future prospective research to use brief cognitive testing as part of predictive/diagnostic models for antipsychotic response and future treatment resistance.

Therefore, this cross-sectional study sought to assess the cognitive profiles of antipsychotic R and NR using the BACS. Based on the existing literature, we hypothesised that TRS patients would have poorer performance across BACS tasks, particularly on verbal memory and verbal fluency measures.
METHODS

\section{Design}

The study used a cross-sectional design comparing antipsychotic treatment $\mathrm{R}$ and antipsychotic NR on cognitive performance.

\section{Setting}

The study was part of 'Schizophrenia: Treatment Resistance and Therapeutic Advances' (STRATA), a consortium which included King's College London (London, UK), University of Manchester (Manchester, UK), Cardiff University (Wales, UK) and University of Edinburgh (Scotland, UK). The aim of the STRATA consortium is to identify neurobiological, cognitive and genetic biomarkers of antipsychotic treatment resistance and NR within schizophrenia and other related psychotic disorders.

\section{Patient and public involvement}

During the early development of the study the views and recommendations of service users and carers regarding the use of stratified medicine research were assessed. Consultations were undertaken with the Institute of Psychiatry, Psychology and Neuroscience's Service User Advisory Group. Service user researchers in London, Manchester and Edinburgh (18 people) carried out focus groups, and one carer focus group in London (8 people). Focus groups were digitally recorded, the transcripts analysed in NVivo V.10 using a simple thematic analysis, and quotations deidentified to protect participants. The results of this research are published in BioMed Central. ${ }^{37}$ Both service users and carers reflected enthusiasm for stratified medicine. Each stage of the study was discussed, including their willingness to participate and attitudes towards, and perceived intrusiveness of different procedures. These individuals also aided in commenting and providing recommendations on consent and participant information forms.

\section{Participants}

One hundred and six participants were recruited following a screening of patients across four sites: King's College London $(\mathrm{N}=38)$, University of Manchester $(\mathrm{N}=32)$, Cardiff University $(\mathrm{N}=16)$ and University of Edinburgh $(\mathrm{N}=18)$. Inclusion criteria were as follows: aged 18-65 years, with a schizophrenia or schizophreniform disorder diagnosis as per Diagnostic and Statistical Manual of Mental Disorders, fifth edition ${ }^{38}$ criteria and be able to read and write English to a sufficient level (see also Egerton et al. ${ }^{39}$ Participants were excluded if they were pregnant, had ever experienced a head injury involving loss of consciousness for more than $5 \mathrm{~min}$, met International Classification of Diseases (ICD) criteria for harmful substance misuse or a psychotic disorder secondary to substance use, scored $<3$ on the CRS (a measure of adherence $)^{4041}$ or had been treated with clozapine in the previous 3 months. All participants gave informed consent prior to enrolment. 


\section{Definition of antipsychotic R and antipsychotic NR}

Participants were defined as antipsychotic treatment $\mathrm{R}$ if they had been treated with only one antipsychotic drug since illness onset or if their antipsychotic drug had been changed only for reasons of adverse effects as opposed to NR. In addition to this, responders needed to have a Clinical Global Impression-Schizophrenia scale (CGI$\mathrm{SCH})^{42}$ of below 4 (moderately ill), a Positive and Negative Syndrome Scale (PANSS) ${ }^{43}$ total score below 60, and a CRS ${ }^{4041}$ level of adherence greater than 3 ('accepts only because compulsory'). Fifty-four treatment responders were recruited into the study.

Antipsychotic treatment NR was defined as having documented treatment with at least two antipsychotics each above the minimum therapeutic dose as defined by the British National Formulary for $>4$ weeks each, a CGISCH severity score of $>3$, a PANSS total severity rating of at least 70, and a CRS adherence score of $>3$. Fifty-two participants met criteria for antipsychotic NR.

\section{MATERIALS \\ Clinical and demographic measures}

Previous and existing drug use were measured using the Alcohol, Drug and Tobacco Inventory. Participants' disorder severity was measured using the MiniInternational Neuropsychiatric Interview (M-Psychotic Disorders; A-Major Depressive Episode; D-Manic/Hypomanic/Bipolar), ${ }^{44}$ Structured Clinical Interview-PANSS (SCI-PANSS) ${ }^{45}$ and CGI-SCH. ${ }^{42}$ Concordance with medication was assessed using the Clinical Rating Scale (CRS) for Schizophrenia. ${ }^{40}{ }^{41}$ Participants also provided demographic data, such as years of previous full-time education, age, gender, as well as information regarding their previous antipsychotic history which were supplemented by medical records.

\section{Measures of cognitive performance}

Cognitive data were collected using the BACS ${ }^{25}$ across all sites at the beginning of the assessment, following the administration of clinical and demographic measures. The battery is designed to take $\sim 30 \mathrm{~min}$ to complete, with minimal training demands, and is designed to be easily administered by clinical and healthcare workers. ${ }^{25}$ The BACS (version A) ${ }^{25}$ consists of six tests from the following cognitive domains: (1) Verbal Memory: List learning task; (2) Working Memory: Digit Sequencing task; (3) Motor Speed: Token motor task; (4) Verbal Fluency: Category instances task (Animals) and phonological (F and S-words); (5) Attention and speed of information processing: Symbol Coding task; (6) Executive Functions: Tower of London task. All tasks on the BACS are scored with higher scores representing better performance. Composite $\mathrm{z}$ and $\mathrm{t}$ scores for the BACS are generated using normative data ${ }^{46}$ and the following formulas: Composite $z$ score $=\frac{\Sigma\left(\Sigma \frac{(\text { raw score-normative score })}{\text { normative standard deviation }}\right)}{3.63}$ with each measure's z score summed and the total divided by 3.63 ; Composite t score $=($ Composite $z$ score $* 10)+50$.

\section{Data analysis}

All analyses were conducted using STATA V.15/SE. ${ }^{47}$ $\chi^{2}$ tests were used to compare cognitive performance across sites in case of site differences. Univariable regressions were used to compare cognitive performance between groups. Multivariable regression analyses were used to adjust univariable results for age, gender and illness duration, due to the reported relationship of age, ${ }^{449}$ gender $^{5051}$ and illness duration ${ }^{5253}$ with cognitive outcomes. Analyses adjusting for anticholinergic effects of antipsychotic medication are presented in online supplemental material (online supplemental table S1).

\section{RESULTS}

Descriptive statistics of demographic and clinical variables between responder groups are reported in table 1 . In the antipsychotic $\mathrm{R}$ group ( $\mathrm{N}=54$ ), four were treated with a first-generation antipsychotic. For the NR group $(\mathrm{N}=52)$, five were treated with a first-generation antipsychotic. All other participants were treated with second-generation antipsychotics.

\section{Cognitive performance}

Mean scores for each group on all BACS tasks and standardised composite scores are displayed in table 2. All measures of the BACS were normally distributed, with exception of the Tower of London task which was moderately negatively skewed (skewness $=-0.95$ ) as per the guidelines from Bulmer. ${ }^{54}$ Cognitive performance on BACS composite and subtests did not significantly differ by site where data were collected.

Univariable linear regression analyses (table 2) observed no significant relationships between response status and BACS performance. Multivariable models adjusted for age, gender and illness duration also observed no significant relationships between response status and cognitive outcomes (table 2).

\section{DISCUSSION}

The present investigation sought to compare specific cognitive deficits in antipsychotic $\mathrm{R}$ and antipsychotic NR using the $\mathrm{BACS}^{25}$ anticipating the greatest deficits for NR in measures of verbal memory and verbal fluency when compared with R. Unlike previous cross-sectional studies, ${ }^{55-62}$ this investigation identified no significant differences in cognitive performance between groups.

Previous cross sectional research investigating differences in cognitive performance between antipsychotic treatment $\mathrm{R}$ and treatment resistant cases have identified poorer performance in verbal, executive function, full-scale IQ cognitive measures, ${ }^{55} 56^{59-61}$ and verbal memory 555606263 in treatment resistant patients. A recent study using a similar methodology and sample size to ours 
Table 1 Demographic and clinical characteristics by group

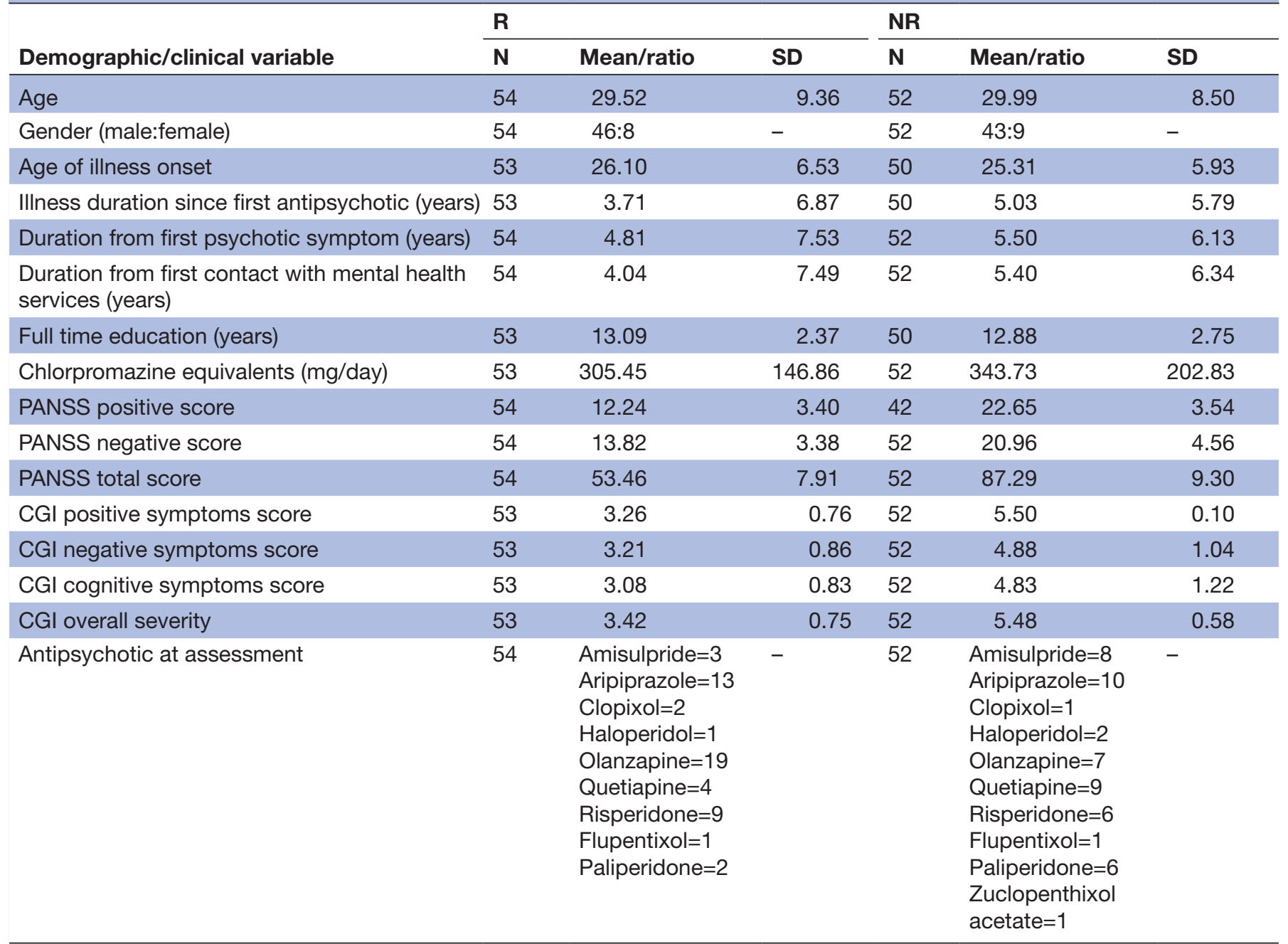

CGI, Clinical Global Impression; NR, antipsychotic non-responder; PANSS, Positive and Negative Syndrome Scale; R, antipsychotic responder.

also failed to show significant differences between antipsychotic R and TRS cases on individual tasks of the BACS ${ }^{64}$ but did observe significant differences on standardised $(\mathrm{z}$ and t) composite scores suggesting overall impairment in the TRS group. Our additional analyses also adjusting for anticholinergic effects (online supplemental table S1) also observed no change to the relationship between BACS and antipsychotic response, suggesting no medication effects on our findings. We also further restricted our analysis to exclude participants that were under dosed (ie, not within the $150-600 \mathrm{mg}$ / per day range) removing 12 participants $(\mathrm{R}=5, \mathrm{NR}=7)$. No change was observed in the pattern of results.

The lack of significant differences in cognitive performance observed between $\mathrm{R}$ and NR groups in our study may be partly explained by the criteria used to define these groups. Unlike earlier investigations, our study did not include clozapine-treated patients, and there may have been less clinical separation between the $\mathrm{R}$ and NR groups than in some previous studies (as discussed in Egerton et al). ${ }^{39}$ Furthermore, in our cross-sectional study design, it is not possible to determine the proportion of participants in the NR group who would meet criteria for TRS. ${ }^{65}$ It is, therefore, possible the NR group was less severely unwell as in some previous studies, which may have reduced the ability to observe potential impairments in cognition due to clinical overlap. Previous investigations which observed group differences in cognitive performance between $\mathrm{R}$ and TRS included patients prescribed clozapine, ${ }^{56} \begin{array}{llll}57 & 59-61 \quad 63 & 64\end{array}$ and reported higher PANSS positive, negative and total scores, 596064 suggesting the NR/TRS groups may have had greater illness severity compared with our sample. Likewise, demographic and clinical variables previously found to be associated with antipsychotic R, such as a younger age and age of illness onset in $\mathrm{NR},{ }^{12-16}$ did not differ between treatment $\mathrm{R}$ and NR in our sample, again suggesting group that compared with previous investigations, there was not enough clinical separation between our samples. In addition, the power calculations for sample size were generated on the basis of being able to provide $>95 \%$ power to detect differences in levels of 
anterior cingulate glutamate ${ }^{39}$ (see Protocol provided in online supplemental material) and it is possible that the sample was underpowered to detect neurocognitive differences using the BACS.

It is also possible that our definition of antipsychotic response and inclusion criteria may have influenced our findings. As per definition, differences were only observed between groups on CGI-SCH and PANSS measures of symptom severity. Psychotic symptoms such as hallucinations, delusions and paranoia (ie, schizophrenia-like symptoms) have been attributed to D2 dopamine receptors and functioning in the striatum, as evidenced by animal models. ${ }^{65}$ It has also been reported that following amphetamine administration, hyperactivity of dopamine transition is associated with the activation of psychotic symptoms. However, amphetamine induced psychosis does not tend to exhibit negative and cognitive symptoms. ${ }^{66}$ In contrast, cognitive deficits in schizophrenia have been reported to be related to functioning in the dorsolateral prefrontal cortex (DLPFC) ${ }^{67} 68$ glutamate to Gamma Aminobutyric Acid (GABA) ratios in the DLPFC, ${ }^{69}$ as well as prefrontal glutamate levels in the dorsal anterior cingulate cortex in antipsychotic-naive patients. ${ }^{69}$ Unlike psychotic symptoms, the Dopamine D1 receptor signalling is essential for cognition. ${ }^{70}$ Therefore, it is possible that the differences in the neurobiological underpinnings between psychotic and cognitive symptoms may also explain why no cognitive differences were observed between groups, as this was biased in favour of psychotic symptoms due to our inclusion criteria.

Another consideration is that our study focused on younger patients early in their treatment trajectories to reduce the potential effects of chronicity and previous medication, with a mean length of treatment of 3-4 years. Most previous cross-sectional investigations also include older samples with a longer duration of illness, ${ }^{5657596064}$ although differences in measures of verbal intelligence and fluency have been quantifiable at the first episode in treatment resistant psychosis. ${ }^{27}$ Trajectory modelling of cognitive performance in first episode psychosis has observed deficits in executive function performance, relative to controls, with these remaining stable over illness duration. ${ }^{71}$ However, deficits in verbal knowledge and memory became more apparent and exaggerated relative to controls following the first episode ${ }^{72}$ Similar exaggerated declines following the first episode have also been observed in measures of verbal memory. ${ }^{71}$ With our sample of patients being early in their treatment, cognitive deficits may have been less marked at this illness stage.

Likewise, this more restricted focus may explain why there was smaller sampling of females in comparison to previous investigations. A recent nationwide cohort study found that on average females are more likely to be first diagnosed with a mood disorder prior to a psychotic diagnosis. ${ }^{74}$ This coupled with the observation that females also tend to have a later onset of psychotic symptoms than males, ${ }^{75}$ it is possible that recruiting younger participants 
may have restricted the true picture of schizophrenia at large within the general population.

Despite not detecting significant differences between antipsychotic R groups, it is worth mentioning the importance of conducting research using clinically transferable measures of cognitive impairment. It may be possible for future researchers to use machines learning algorithms to identify subgroups of schizophrenia from cognitive outcomes. Bak et $a l^{76}$ used Gaussian mixture modelling to identify two distinct subgroups in antipsychotic-naive first episode schizophrenia samples. In this study, cognitive and electrophysiological data were used to identify the two groups. When predicting treatment response, assessed by the PANSS, there was a significant predictive relationship between group and antipsychotic response. Therefore, future research should aim to use more machine learning techniques to identify patterns of cognitive performance within schizophrenia subsamples and investigate antipsychotic response between these groups.

\section{CONCLUSIONS}

Within this cross-sectional investigation, we observed no differences in cognitive performance between antipsychotic R and NR. This may be because there was less clinical separation between these groups in our sample in comparison to previous investigations. Future investigations should consider the role of machine learning techniques to investigate the role of cognitive functions in identifying subgroups of schizophrenia using first episode cohorts and how this may differ in future stages of treatment resistance. Such research using antipsychotic-naïve patients vs healthy controls has observed strong group discrimination using cognitive measures in comparison to electrophysiology and MRI methods, ${ }^{77}$ with other investigations observing distinct subgroups in schizophrenia from differences in early information processing and higher cognitive functions. ${ }^{74}$

\section{Author affiliations}

'Department of Psychosis Studies, King's College London Institute of Psychiatry Psychology and Neuroscience, London, UK

${ }^{2}$ NIHR Biomedical Research Centre at South London and Maudsley NHS Foundation Trust and King's College London, London, UK

${ }^{3}$ Division of Psychology and Mental Health, The University of Manchester, Manchester, UK

${ }^{4}$ Greater Manchester Mental Health NHS Foundation Trust, Manchester, UK ${ }^{5}$ Psychiatry, The University of Edinburgh Division of Psychiatry, Edinburgh, UK ${ }^{6}$ MRC Centre for Neuropsychiatric Genetics and Genomics, Cardiff University, Cardiff, UK

Contributors JM, RMM, ODH, AE, EK, RD, LK, JD, AM, TC, JL, CS-P, JW, BD and SLa contributed to the design and implementation of the study. EM completed analyses and wrote the manuscript with the assistance of JM and EK. JM, RMM, ODH, AE, EK, SLe, JD, SLa provided comments on the manuscript. JM is the guarantor.

Funding STRATA is funded by a grant from the Medical Research Council (MRC) to $\mathrm{JM}$, grant reference MR/L011794. EM's PhD is funded by the MRC-doctoral training partnership studentship in Biomedical Sciences at King's College London. JM, EK, RMM, AE and ODH are part funded by the National Institute for Health Research (NIHR) Biomedical Research Centre at South London and Maudsley NHS Foundation
Trust and King's College London. In the past three years, SLe has received personal support from Sunovion.

Disclaimer The views expressed are those of the authors and not necessarily those of the NHS, the MRC, the NIHR, Sunovion or the Department of Health.

Competing interests None declared.

Patient consent for publication Not applicable.

Ethics approval This study was approved by the South East Coast-Surrey Research Ethics Committee; REC: 15/LO/0038. All participants provided informed consent prior to participation.

Provenance and peer review Not commissioned; externally peer reviewed.

Data availability statement Data are available on reasonable request.

Supplemental material This content has been supplied by the author(s). It has not been vetted by BMJ Publishing Group Limited (BMJ) and may not have been peer-reviewed. Any opinions or recommendations discussed are solely those of the author(s) and are not endorsed by BMJ. BMJ disclaims all liability and responsibility arising from any reliance placed on the content. Where the content includes any translated material, BMJ does not warrant the accuracy and reliability of the translations (including but not limited to local regulations, clinical guidelines, terminology, drug names and drug dosages), and is not responsible for any error and/or omissions arising from translation and adaptation or otherwise.

Open access This is an open access article distributed in accordance with the Creative Commons Attribution 4.0 Unported (CC BY 4.0) license, which permits others to copy, redistribute, remix, transform and build upon this work for any purpose, provided the original work is properly cited, a link to the licence is given, and indication of whether changes were made. See: https://creativecommons.org/ licenses/by/4.0\%.

ORCID iDs

Edward Millgate http://orcid.org/0000-0001-5424-8261

Richard Drake http://orcid.org/0000-0003-0220-4835

\section{REFERENCES}

1 National Institute for Health and Care Excellence. National collaborating centre for mental health (UK. psychosis and schizophrenia in adults: treatment and management) 2014.

2 Elkis H. Treatment-resistant schizophrenia. Psychiatric Clinics of North America 2007;30:511-33.

3 Demjaha A, Lappin JM, Stahl D, et al. Antipsychotic treatment resistance in first-episode psychosis: prevalence, subtypes and predictors. Psychol Med 2017;47:1981-9.

4 Kane JM, Agid O, Baldwin ML, et al. Clinical guidance on the identification and management of treatment-resistant schizophrenia. J Clin Psychiatry 2019;80 doi:10.4088/JCP.18com12123

5 Üçok A, Çıkrıkçılı U, Ergül C, et al. Correlates of clozapine use after a first episode of schizophrenia: results from a long-term prospective study. CNS Drugs 2016;30:997-1006.

6 Köhler-Forsberg O, Horsdal HT, Legge SE, et al. Predictors of nonhospitalization and functional response in clozapine treatment: a nationwide, population-based cohort study. J Clin Psychopharmacol 2017;37:148-54.

7 Yoshimura B, Yada Y, So R, et al. The critical treatment window of clozapine in treatment-resistant schizophrenia: secondary analysis of an observational study. Psychiatry Res 2017;250:65-70.

8 Griffiths K, Millgate E, Egerton A, et al. Demographic and clinical variables associated with response to clozapine in schizophrenia: a systematic review and meta-analysis. Psychol Med 2021;51:376-86.

9 Lally J, Gaughran F, Timms P, et al. Treatment-resistant schizophrenia: current insights on the pharmacogenomics of antipsychotics. Pharmgenomics Pers Med 2016;9:117.

10 Gillespie AL, Samanaite R, Mill J, et al. Is treatment-resistant schizophrenia categorically distinct from treatment-responsive schizophrenia? A systematic review. BMC Psychiatry 2017;17:1-4.

11 Lally J, Ajnakina O, Di Forti M, et al. Two distinct patterns of treatment resistance: clinical predictors of treatment resistance in first-episode schizophrenia spectrum psychoses. Psychol Med 2016;46:3231-40.

12 Wimberley T, Støvring H, Sørensen HJ, et al. Predictors of treatment resistance in patients with schizophrenia: a population-based cohort study. Lancet Psychiatry 2016;3:358-66.

13 Smart SE, Kępińska AP, Murray RM, et al. Predictors of treatment resistant schizophrenia: a systematic review of prospective observational studies. Psychol Med 2021;51:44-53. 
14 Legge SE, Dennison CA, Pardiñas AF, et al. Clinical indicators of treatment-resistant psychosis. Br J Psychiatry 2020;216:259-66.

15 Bozzatello P, Bellino S, Rocca P. Predictive factors of treatment resistance in first episode of psychosis: a systematic review. Front Psychiatry 2019;10:67.

16 Smart S. Clinical and genetic predictors of treatment resistant psychosis (Doctoral dissertation, King's College London) 2020.

17 Toulopoulouand T, Murray RM. Verbal memory deficit in patients with schizophrenia: an important future target for treatment. Expert Rev Neurother 2004;4:43-52.

18 Fatouros-Bergman H, Cervenka S, Flyckt L, et al. Meta-analysis of cognitive performance in drug-naïve patients with schizophrenia. Schizophr Res 2014;158:156-62.

19 Mesholam-Gately RI, Giuliano AJ, Goff KP, et al. Neurocognition in first-episode schizophrenia: a meta-analytic review. Neuropsychology 2009;23:315.

20 Kravariti E, Morgan K, Fearon P, et al. Neuropsychological functioning in first-episode schizophrenia. Br J Psychiatry 2009;195:336-45.

21 Snitz BE, MacDonald III AW, Carter CS. Cognitive deficits in unaffected first-degree relatives of schizophrenia patients: a metaanalytic review of putative endophenotypes.

22 Bora E, Özerdem A. Meta-analysis of longitudinal studies of cognition in bipolar disorder: comparison with healthy controls and schizophrenia. Psychol Med 2017;47:2753-66.

23 Fagerlund B, Pantelis C, Jepsen JR. Differential effects of age at illness onset on verbal memory functions in antipsychotic-naïve schizophrenia patients aged 12-43 years. Psychol Med 2020;11.

24 Millgate E, Hide O, Lawrie S. Neuropsychological differences between treatment-resistant and treatment-responsive schizophrenia: a systematic review \& meta-analysis. [Manuscript submitted for publication]. Institute of Psychiatry, Psychology and Neuroscience, King's College London.

25 Keefe RSE, Goldberg TE, Harvey PD, et al. The brief assessment of cognition in schizophrenia: reliability, sensitivity, and comparison with a standard neurocognitive battery. Schizophr Res 2004;68:283-97.

26 Velligan DI, Bow-Thomas CC. Executive function in schizophrenia. Semin Clin Neuropsychiatry 1999;4:24-33.

27 Kravariti E, Dixon T, Frith C, et al. Association of symptoms and executive function in schizophrenia and bipolar disorder. Schizophr Res 2005;74:221-31.

28 Lee J, Park S. Working memory impairments in schizophrenia: a meta-analysis. J Abnorm Psychol 2005;114:599.

29 Forbes NF, Carrick LA, Mclntosh AM, et al. Working memory in schizophrenia: a meta-analysis. Psychol Med 2009;39:889.

30 Flashman LA, Flaum M, Gupta S, et al. Soft signs and neuropsychological performance in schizophrenia. Am J Psychiatry 1996;153:526-32.

31 Bruder GE, Wexler BE, Sage MM, et al. Verbal memory in schizophrenia: additional evidence of subtypes having different cognitive deficits. Schizophr Res 2004;68:137-47.

32 Hill SK, Beers SR, Kmiec JA, et al. Impairment of verbal memory and learning in antipsychotic-naïve patients with first-episode schizophrenia. Schizophr Res 2004;68:127-36

33 Bokat CE, Goldberg TE. Letter and category fluency in schizophrenic patients: a meta-analysis. Schizophr Res 2003;64:73-8.

34 Ojeda N, Sánchez P, Peña J, et al. Verbal fluency in schizophrenia: does cognitive performance reflect the same underlying mechanisms in patients and healthy controls? J Nerv Ment Dis 2010;198:286-91.

35 Harris JG, Minassian A, Perry W. Stability of attention deficits in schizophrenia. Schizophr Res 2007;91:107-11.

36 Carter JD, Bizzell J, Kim C, et al. Attention deficits in schizophreniapreliminary evidence of dissociable transient and sustained deficits. Schizophr Res 2010;122:104-12.

37 Rose D, Papoulias C, MacCabe J, et al. Service users' and carers' views on research towards stratified medicine in psychiatry: a qualitative study. BMC Res Notes 2015;8:1-9.

38 American Psychiatric Association. Diagnostic and statistical manual of mental disorders (DSM-5尺). American Psychiatric, 2013.

39 Egerton A, Murphy A, Donocik J. Dopamine and glutamate in Antipsychotic-Responsive compared with AntipsychoticNonresponsive psychosis: a multicenter positron emission tomography and magnetic resonance spectroscopy study (strata). Schizophrenia Bulletin 2020.

40 Kemp R, Hayward P, Applewhaite G, et al. Compliance therapy in psychotic patients: randomised controlled trial. BMJ 1996;312:345-9.

41 Kemp R, Kirov G, Everitt B, et al. Randomised controlled trial of compliance therapy. 18-month follow-up. Br J Psychiatry 1998;172:413-9.

42 Guy W. ECDEU assessment manual for psychopharmacology. US department of health, education, and welfare, public health service, alcohol, drug abuse, and mental health administration, National Institute of mental health, psychopharmacology research branch, division of extramural research programs 1976

43 Kay SR, Fiszbein A, Opler LA. The positive and negative syndrome scale (PANSS) for schizophrenia. Schizophr Bull 1987;13:261.

44 Sheehan DV, Lecrubier Y, Sheehan KH, et al. The Mini-International neuropsychiatric interview (MINI): the development and validation of a structured diagnostic psychiatric interview for DSM-IV and ICD-10. J Clin Psychiatry 1998;59:22-33.

45 Opler LA, Kay SR, Lindenmayer JP. Structured clinical interview: the positive and negative syndrome scale (SCI-PANSS). North Tonawanda, NY: Multi-Health Systems, 1999.

46 Keefe RSE, Harvey PD, Goldberg TE, et al. Norms and standardization of the brief assessment of cognition in schizophrenia (BACs). Schizophr Res 2008;102:108-15.

47 StataCorp LL. Stata statistical software: release 15 College Station, TX, 2017. Available: www. stata. com/features/documentation/ [Accessed 1 Mar 2018].

48 Craik FIM, Bialystok E. Cognition through the lifespan: mechanisms of change. Trends Cogn Sci 2006;10:131-8.

49 Harvey PD. What is the evidence for changes in cognition and functioning over the lifespan in patients with schizophrenia? J Clin Psychiatry 2014;75:34-8.

50 Perlick D, Mattis S, Stastny P, et al. Gender differences in cognition in schizophrenia. Schizophr Res 1992;8:69-73.

51 Han M, Huang X-F, Chen DC, et al. Gender differences in cognitive function of patients with chronic schizophrenia. Prog Neuropsychopharmacol Biol Psychiatry 2012;39:358-63.

52 Cuesta MJ, Peralta V, Zarzuela A. Illness duration and neuropsychological impairments in schizophrenia. Schizophr Res 1998;33:141-50. Oct.

53 Bagney A, Rodriguez-Jimenez R, Martinez-Gras I, et al. Negative symptoms and executive function in schizophrenia: does their relationship change with illness duration? Psychopathology 2013;46:241-8.

54 Bulmer MG. Principles of statistics. Courier Corporation, 1979.

55 Lawrie SM, Ingle GT, Santosh CG, et al. Magnetic resonance imaging and single photon emission tomography in treatment-responsive and treatment-resistant schizophrenia. Br J Psychiatry 1995;167:202-10.

56 Smith RC, Kadewari RP, Rosenberger JR, et al. Nonresponding schizophrenia: differentiation by neurological soft signs and neuropsychological tests. Schizophr Bull 1999;25:813-25.

57 Joober R, Rouleau GA, Lal S, et al. Neuropsychological impairments in neuroleptic-responder vs. -nonresponder schizophrenic patients and healthy volunteers. Schizophr Res 2002;53:229-38.

58 de Bartolomeis A, Balletta R, Giordano S, et al. Differential cognitive performances between schizophrenic responders and nonresponders to antipsychotics: correlation with course of the illness, psychopathology, attitude to the treatment and antipsychotics doses. Psychiatry Res 2013;210:387-95.

59 Bourque J, Lakis N, Champagne J, et al. Clozapine and visuospatial processing in treatment-resistant schizophrenia. Cogn Neuropsychiatry 2013;18:615-30.

60 Frydecka D, Beszłej JA, Gościmski P, et al. Profiling cognitive impairment in treatment-resistant schizophrenia patients. Psychiatry Res 2016;235:133-8.

61 Kravariti E, Demjaha A, Zanelli J, et al. Neuropsychological function at first episode in treatment-resistant psychosis: findings from the ÆESOP-10 study. Psychol Med 2019;49:2100-10.

62 Huang J, Zhu Y, Fan F, et al. Hippocampus and cognitive domain deficits in treatment-resistant schizophrenia: a comparison with matched treatment-responsive patients and healthy

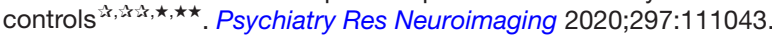

63 lasevoli F, Avagliano C, Altavilla B, et al. Evaluation of a few discrete clinical markers may predict categorization of actively symptomatic non-acute schizophrenia patients as treatment resistant or responders: a study by ROC curve analysis and multivariate analyses. Psychiatry Res 2018;269:481-93.

64 Sosin D, Ivashchenko D, Sozaeva Z, et al. Cognitive impairment in patients with treatment resistant schizophrenia: associations with DRD2, DRD3, HTR2A, BDNF and CYP2D6 genetic polymorphisms. Neurol Psych Brain Res 2019;33:48-55.

65 Kellendonk C, Simpson EH, Polan HJ, et al. Transient and selective overexpression of dopamine D2 receptors in the striatum causes persistent abnormalities in prefrontal cortex functioning. Neuron 2006;49:603-15.

66 Voce A, Calabria B, Burns R, et al. A systematic review of the symptom profile and course of methamphetamine-associated psychosis. Subst Use Misuse 2019;54:549-59.

67 Miller EK, Cohen JD. An integrative theory of prefrontal cortex function. Annu Rev Neurosci 2001;24:167-202. 
68 Schoonover KE, Dienel SJ, Lewis DA. Prefrontal cortical alterations of glutamate and GABA neurotransmission in schizophrenia: insights for rational biomarker development. Biomark Neuropsychiatry 2020;3:100015.

69 Bojesen KB, Broberg BV, Fagerlund B, et al. Associations between cognitive function and levels of glutamatergic metabolites and gamma-aminobutyric acid in antipsychotic-naïve patients with schizophrenia or psychosis. Biol Psychiatry 2021;89:278-87.

70 Arnsten AF. Catecholamine modulation of prefrontal cortical cognitive function. Trends Cogn Sci 1998;2:436-47.

71 Chan SK, Chan HY, Honer WG. Predictors of treatment-resistant and Clozapine-Resistant schizophrenia: a 12-year follow-up study of First-Episode Schizophrenia-Spectrum disorders. Schizophrenia Bulletin 2020.

72 Zanelli J, Mollon J, Sandin S, et al. Cognitive change in schizophrenia and other psychoses in the decade following the first episode. Am J Psychiatry 2019;176:811-9.
73 Barder HE, Sundet K, Rund BR, et al. Ten year neurocognitive trajectories in first-episode psychosis. Front Hum Neurosci 2013;7:643.

74 Sommer IE, Tiihonen J, van Mourik A, et al. The clinical course of schizophrenia in women and men-a nation-wide cohort study. NPJ schizophrenia 2020;6:1-7.

75 Ochoa S, Usall J, Cobo J, et al. Gender differences in schizophrenia and first-episode psychosis: a comprehensive literature review. Schizophr Res Treatment 2012;2012:1-9.

76 Bak N, Ebdrup BH, Oranje B, et al. Two subgroups of antipsychoticnaive, first-episode schizophrenia patients identified with a Gaussian mixture model on cognition and electrophysiology. Transl Psychiatry 2017;7:e1087.

77 Ebdrup BH, Axelsen MC, Bak N, et al. Accuracy of diagnostic classification algorithms using cognitive-, electrophysiological-, and neuroanatomical data in antipsychotic-naïve schizophrenia patients. Psychol Med 2019;49:2754-63. 